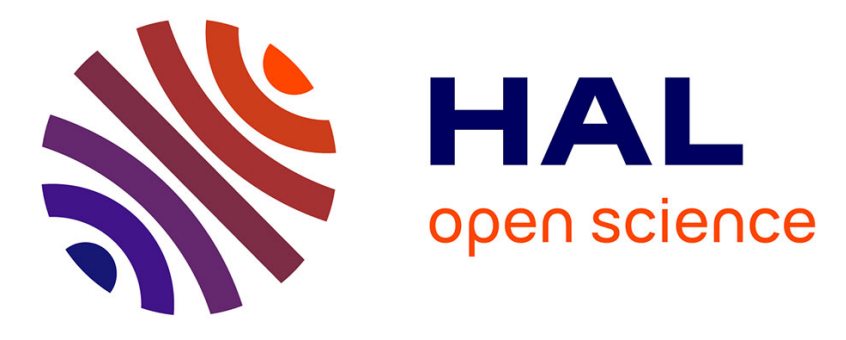

\title{
Integrating Physical and Molecular Insights on Immune Cell Migration
}

\author{
Hélène Moreau, Matthieu Piel, Raphael Voituriez, Ana-Maria
}

Lennon-Duménil

\section{- To cite this version:}

Hélène Moreau, Matthieu Piel, Raphael Voituriez, Ana-Maria Lennon-Duménil. Integrating Physical and Molecular Insights on Immune Cell Migration. Trends in Immunology, 2018, 10.1016/j.it.2018.04.007 . hal-01840086

\section{HAL Id: hal-01840086 \\ https: / hal.sorbonne-universite.fr/hal-01840086}

Submitted on 16 Jul 2018

HAL is a multi-disciplinary open access archive for the deposit and dissemination of scientific research documents, whether they are published or not. The documents may come from teaching and research institutions in France or abroad, or from public or private research centers.
L'archive ouverte pluridisciplinaire HAL, est destinée au dépôt et à la diffusion de documents scientifiques de niveau recherche, publiés ou non, émanant des établissements d'enseignement et de recherche français ou étrangers, des laboratoires publics ou privés. 


\section{Integrating physical and molecular insights of immune cell migration}

Hélène D. Moreau ${ }^{1 *}$, Matthieu Piel ${ }^{2,3}$, Raphaël Voituriez $^{4}$, Ana-Maria Lennon-Duménil ${ }^{1 *}$

\section{Affiliations}

${ }^{1}$ INSERM U932, Institut Curie, ANR-10-IDEX-0001-02 PSL* and ANR-11-LABX-0043, Paris, France. ${ }^{2}$ Institut Curie, PSL Research University, CNRS, UMR 144, F-75005, Paris, France. ${ }^{3}$ Institut Pierre-Gilles de Gennes, PSL Research University, F-75005, Paris, France.

${ }^{4}$ Laboratoire Jean Perrin, UM 8237 CNRS/UPMC, 4 place Jussieu 75005 Paris.

*Correspondence: Helene.moreau@curie.fr (HD Moreau); Ana-Maria.Lennon@curie.fr (A.M. Lennon-Duménil)

Keywords: Cell migration, cytoskeleton, microenvironment, biophysics 


\section{Abstract}

The function of most immune cells depends on their ability to migrate through complex microenvironments, either randomly to patrol for the presence of antigens, or directionally to reach their next site of action. The actin cytoskeleton and its partners are key conductors of immune cell migration as they control the intrinsic migratory properties of leukocytes as well as their capacity to respond to cues present in their environment. In this review, we will focus on the latest discoveries regarding the role of the actomyosin cytoskeleton in optimizing immune cell migration in complex environments, with a special focus on recent insights provided by physical modeling.

\section{TRENDS}

- Immune cells in vivo mostly use non-adherent confined migration. This type of migration relies on Myosin II-dependent actin retrograde flow, which generates forward propulsion forces by friction with the environment, rather than through specific receptor-ligand interactions.

- An alternation of fast and slow migration conducted by distinct actin subcellular pools is observed in many immune cells. Such intermittent migration mode can optimize space exploration by immune cells.

- Immune cell migration is guided by a combination of chemical and physical environmental cues. The actin cytoskeleton contributes to detection and response to these cues, allowing immune cells to integrate them and navigate in complex environments. 


\section{Effective immune responses rely on optimized cell migration}

In mammals, the adaptive immune response results from the collective effort of distinct cell populations that circulate between tissues and/or lymphoid organs. While detection of danger often occurs in peripheral tissues, i.e. in the sites that are more often exposed to external threats such as the skin and mucosa, initiation of the adaptive immune response primarily takes place in lymphoid organs. Multiple immune players are at work: leukocytes such as neutrophils and monocytes directly migrate to the site of infection or damage to provide a rapid defense, while tissue-resident dendritic cells (DCs) that detect danger at the periphery transport the information to lymph-nodes for presentation to $\mathrm{T}$ lymphocytes, which in turn interact with B lymphocytes for full adaptive immunity activation. It therefore appears that the success of the immune response relies on the ability of these various cell populations to be at the right place at the right time to efficiently perform their immune function. How is their migration optimized to ensure immune efficacy? We will focus our review on this question and present recent evidence highlighting how the actomyosin cytoskeleton helps leukocytes adapt their migration mode to their functional requirement in time and space, as well as to the tremendous physical complexity of the tissues they navigate through. We will further emphasize how physical modeling has helped immunologists move forward in the unraveling of the basic ingredients that account for leukocyte locomotion. 


\section{THE ACTIN CYTOSKELETON IN FORCE GENERATION AND CELL MIGRATION}

\section{Basics of actin-dependent cell migration: forces and adhesion/friction.}

Beyond the case of immune cells, defining the basic mechanisms of eukaryotic cell migration in general has been a major challenge. Besides the indisputable progress made by cell biology and biochemistry to identify the molecular players involved, the contribution of physical modeling to the field has become very important over the last decade [1-3]. Basic physical laws show that motion at the scale of the cell can occur only under two main requirements: (1) force generation and (2) momentum transfer to the environment, which implies a contact interaction with the cell, for example through adhesion or friction (Figure 1). At the molecular scale, mechanical forces involved in cell migration are in most of cases produced by the actomyosin system, which is extremely conserved in the eukaryotic world. We refer to this system as an "active system" because it converts the energy of ATP hydrolysis into mechanical work. Conversion occurs through two main mechanisms. First, the polymerization of actin filaments, which generates forces by pushing against the cell membrane, for example in cell protrusions such as lamellipodia or filopodia. Second, the active crosslinking of actin filaments by molecular motors, in particular myosin II, which exerts contractile stresses on the actin network. Regarding momentum transfer, it has been classically attributed to adhesion complexes such as focal adhesions in fibroblasts or podosomes in immune cells, which involve transmembrane proteins from the integrin family. In this picture, a migrating cell is characterized by an adherent protrusive actin network at the cell front, which pushes the leading edge forward, and a contractile actomyosin network at the back that drags the cell rear. If these elementary molecular mechanisms are now fairly well characterized, their global 
integration and coordination at the cell scale is however not fully understood, and motile phenotypes can vary significantly between types of cells and microenvironments.

\section{Immune cells in confinement undergo actin-dependent non-adhesive cell migration}

Traditionally, cells relying on strong adhesion complexes and pronounced protrusion activity at their leading edge are classified as belonging to the mesenchymal migration mode, while less adherent cells with a roundish phenotype and higher contractile capacity belong to the amoeboid mode[4]. Following this classification, immune cells such as DCs, which lack focal adhesions and migrate in a myosin II dependent manner, display an amoeboid phenotype. However, this standard classification of migration modes, which tends to assign to each cell type a specific migration mechanism has been challenged over the recent years by different studies that showed that the physical properties of the cell environment - geometric confinement and adhesion - could significantly change the motile phenotypes of a given cell type $[5,6]$. In particular, it was already observed in vitro by Malawista \& de Boisfleury [7] that leukocytes with impaired adherence could migrate only if confined between two flat surfaces (i.e. a 2D slab geometry, see Figure 1). More recently, it was demonstrated that activated DCs migrate in peripheral and lymphoid tissues, or in vitro in collagen gels, independently of integrins, while in vitro migration on flat 2D substrates remains adhesiondependent $[8,9]$. These results pointed to an important role of the local geometry of the cellular environment. In fact, geometric confinement was shown to be crucial to allow nonadhesive migration in experiments using 1D channels or 2D slabs, where geometric confinement was controlled by microfabrication techniques and adhesion varied by chemical treatment of surfaces $[10,11]$. These experiments showed that even after a non-specific chemical suppression of adhesion (for example by PEG coating), which prevented most specific cell substrate interaction on flat 2D surfaces, motile behaviors could be observed in 
confinement. Interestingly, these findings were shown to go beyond immune cells and a similar non-adhesive migration mode in confinement was observed in various cell types, including those classically labelled as mesenchymal in classical 2D adhesive migration assays $[10,12]$. Overall these results indicate that non-adhesive migration in confinement is a very robust migration mode, which is especially relevant to immune cells such as DCs, whose function in vivo is to patrol in the complex and intrinsically confined geometry of interstitial tissues. This does not rule out adhesion-based locomotion for immune cells, which can be required for certain steps such as transmigration, as well as in looser tissues [13]. It rather suggests that immune cells that have to navigate through potentially very diverse tissue geometries could benefit from migration mode plasticity.

The analysis of non-adhesive motile immune cells in confinement revealed a very conserved phenotype, which displays the prototypical shape of migrating neutrophils with a pronounced uropod and a smooth, rounded front. Importantly, it is characterized by a cell-scale retrograde flow of cortical actin, which is critically driven by the contractile rear [9-12]. This retrograde actin flow, driven by a gradient of contractile stress in the actin network, is at the core of adhesion-independent migration: force transmission to the substrate is then mediated by a non-specific effective friction that results from the indirect interaction (involving either transmembrane proteins or lipids) of flowing actin filaments and extracellular matrix (ECM) molecules, and does not require specific adhesion complexes. Indeed, unspecific friction can be generated by leukocyte surface molecules, which may contain low affinity binding sites for extracellular proteoglycans, collagens or laminins. They may vary in composition and therefore in the "molecular" friction they impose on the motile leukocyte. Friction forces may also result from the microvilli or veiled structures present at the surface of some leukocytes such as neutrophils, DCs or T cells [14], which provide what we could call "geometrical" or 
"structural" friction. Importantly, such non-specific friction is drastically increased in confinement due to a higher pressure, which can yield sufficient propulsion forces even on slippery surfaces such as PEGylated surfaces $[10,11,15]$. This mechanism was originally called chimneying $[7,16]$. Interestingly, it was shown [9] that in DCs, the actin flow speed is significantly increased in non-adherent conditions to compensate the lack of adhesion and overall maintain the cell speed. These main features are recapitulated by minimal physical models based on active gel theory [3, 11, 17-19], and even seem reproducible in reconstituted systems [20], which pleads for the robustness of non-adherent confined migration in immune cells and beyond. At larger scales, it was argued that cell scale actin flows observed in nonadherent migration stabilized cell polarity, thus leading to more persistent cell trajectories and potentially a more efficient exploration of space [17].

\section{ACTIN DYNAMICS OPTIMIZE IMMUNE CELL TRAJECTORIES AND FUNCTION}

From the first part of this review, we learned that the actin cytoskeleton controls both the generation of forces and the transfer of momentum to serve the motility of immune cells. In this second part, we will discuss how actin dynamics in migrating leukocytes determine the type of trajectory they display when evolving in complex environments and accordingly, influence their immune function.

\section{Actin dynamics determine the migratory pattern of immune cells}

\section{Through regulation of actin nucleation}

Analysis of actin dynamics in migrating DCs and T lymphocytes has revealed that their migratory behavior is often adapted to their immune function. Such adaptation is particularly evident when analyzing the migration modes of immature and mature DCs. While immature 
DCs, whose main function is tissue patrolling, alternate phases of slow and fast migration, mature DCs, which are in charge of transporting antigens to lymph nodes to initiate the immune response, migrate at a rather constant high speed and with a higher persistence [21]. By investigating actin dynamics in these cells, two separate actin pools were identified as accounting for these different migratory patterns. At the cell rear, actin cables nucleated by the formin mDial downstream of the RhoA small GTPase are responsible for fast and persistent migration, both in immature and mature DCs. The second actin pool identified is located at the front of immature DCs, mostly in ruffles and around macropinosomes, and depends on nucleation of branched actin by the Arp2/3 complex downstream of the small GTPase Cdc42. It contributes to antigen capture but reduces cell velocity in immature DCs. The Cdc42-Arp2/3 axis is inactivated upon DC maturation, leading to the loss of actin at the cell front, which has two consequences: (1) it down-regulates antigen capture and (2), together with CCR7 upregulation, it allows DCs to migrate in a faster and more directional manner towards lymphatic vessels and lymph nodes. Consistent with these findings, it was shown that the deletion of the Hem1 subunit of WAVE, the Nucleating Promoting Factor (NPF) that activates Arp2/3 at the leading edge, leads to a loss of lamellipodia in immature DCs [22]. They then adopt a needle-like shape that depends on F-actin nucleation by formins. The effect of WAVE deletion is strikingly different on mature DCs, which present a rounded cell body but numerous dynamic filopodia that extends in multiple directions. These results point toward a differential regulation of actin nucleation by Arp $2 / 3$ and formins, depending on the state of the cell and leading to distinct intrinsic migratory properties. Interestingly, similar observations have been made in neutrophils [23] as well as in T lymphocytes, in which mDia1 is required for migration $[24,25]$ while Arp2/3 is not [26]. In agreement with these findings, Arp2/3 activity is mainly required for Integrin-dependent cellular functions of macrophages in vivo, this complex being dispensable for the locomotion of these cells in 3-dimensional 
environments [27]. It therefore appears that most leukocytes do not rely on branched actin nucleation for migration in confinement, consistent with their migration being independent of adhesion in such complex environment.

\section{Through regulation of actin-based molecular motors}

Actin-based molecular motors, which regulate the dynamics of the actin cytoskeleton, further play a key role in determining the migration mode of leukocytes. This particularly applies to DCs: the arrest phases observed when Arp2/3 nucleates actin at the front of immature DCs are associated to the cell front recruitment of myosin II, which results in its depletion from the cell rear [28]. Strikingly, myosin II local inhibition at the front of these cells is sufficient to increase their speed. These transient enrichments of myosin II at the front of immature DCs not only decrease their speed but further help the formation and maturation of macropinosomes, i.e. the arrival of their content to lysosomes, where they are processed for presentation onto MHC molecules. Consistent with these findings, periodic recruitment of myosin II at the cell front is mediated by its interaction with the MHC class II-associated Invariant Chain (Ii or CD74) [29]. We proposed that diversion of myosin II by Ii from the migratory apparatus at the rear of immature DCs to their front allows the coordination of antigen capture and cell migration. Accordingly, mature DCs, which neither nucleate actin nor recruit myosin II at their front migrate in a fast and continuous manner, similarly to Iideficient immature DCs $[21,29]$. Consistent with the idea that migration of cells under confinement relies on an actin retrograde flow induced by an increased contractility at the rear of the cell (See first part), the recruitment of myosin II at the front of the cell would perturb the gradient of contractility and as a result would decrease actin flow and thus cell speed [15]. 
Physical modeling of the competition for myosin II of the cell rear and the cell front indicates that it generates a dynamic instability whereby cells switch from unidirectional persistent migration to self-oscillation [30], suggesting that this will apply to any cell wherein there is competition for limited resources involved in locomotion. Of note, B and T lymphocytes also use myosin II to migrate in confinement [29, 31]. Interestingly, arrest phases upon TCR activation require the down-regulation of myosin II activity [32]. Similarly, in activated natural killer (NK) cells, myosin II is needed for granule transport towards the target cell [33]. Altogether, these results suggest that a similar scenario might be operating in these cells: myosin II enrichment at their rear promotes their migration and its diversion to the front in response to antigen receptor stimulation helps these cells reducing their speed while forming an immune synapse with their antigen presenting (B and T lymphocytes) or target (NK cells) cell $[34,35]$. In vivo imaging of myosin II at the single cell level should further substantiate this paradigm, according to which diversion of myosin II from the front to the back and viceversa would be central in coordinating immune cell migration to other immune cell functions. Another interesting actin-based motor protein that has been shown to modify the migration modes of T lymphocytes is the myosin IG [36]. This is a non-classical calcium-responsive myosin that binds to membrane-associated lipids such as $\mathrm{PI}(4,5) \mathrm{P}_{2}$ through their tail and to actin through their head. By linking the actin cytoskeleton to the plasma membranes, class I myosins have been shown to be major regulators of lymphocyte membrane tension [36, 37]. Noticeably, myosin IG-deficient $\mathrm{T}$ cells migrate faster and change direction less often that their wild-type counterpart, suggesting that phases of arrest are facilitated by this motor protein and might thus require a tight regulation of surface tension. Whether myosins I also control the locomotion modes of immature and mature DCs remain to be investigated. 


\section{Intermittent migration: an internal clock?}

The function of many immune cells first depends on their capacity to find a needle in the haystack of the tissue environment. For example, immature DCs need to efficiently patrol tissues to detect rare danger-associated antigens, while naïve $\mathrm{T}$ cells need to sample many APCs before potentially finding the one presenting its cognate antigen in a lymph node. Here, we will present recent experimental and theoretical evidence suggesting that by imposing specific migration modes to leukocytes, the actin cytoskeleton contributes to the optimization of their trajectories and immune function.

As mentioned above, immature DCs alternate fast and slow motility phases depending on the subcellular localization of their actomyosin cytoskeleton: when myosin II contracts actin at the DC rear, it triggers forward movement, whereas it reduces migration but allows antigen capture when it contracts actin at the cell front. Interestingly, alternating fast migration with limited environment sampling and slow migration with high antigen uptake can optimize the search for hard to find targets in a large space, and is referred to as an "intermittent random walk" (See Textbox). The duration of the migration periods in immature DCs lies close to the optimal values determined by theoretical modeling [28], suggesting that the internal clock defined by the regulation of the actomyosin cytoskeleton has evolved to optimize the sampling of the environment by these cells. The use of an intermittent migration mode might thus help immature DCs finding rare target antigens (separated by more than $800 \mu \mathrm{m}$ ), explaining how they efficiently perform their immune function despite their low concentration in peripheral tissues.

In vivo, T cells were also found to alternate phases of high and low motility [38]. However, given the complex environment of tissues, these observations cannot assess whether this stop and go behavior is an intrinsic property of $\mathrm{T}$ cells or whether it is environmentally driven. Modeling the "stop and go" behavior of T cells has resulted in distinct interpretations. It could 
be a consequence of an internal clock that would make $\mathrm{T}$ cells adopt a Levy-walk search strategy (See Textbox) as was described for migration in the infected brain [39]. Alternatively, it can be explained by the encounter of obstacles in the lymph node during random constant migration [40] and/or by opposite chemotactic go signals and TCRdependent stop signals that $\mathrm{T}$ cells integrate while migrating [41]. Interestingly, in microchannels, contrary to immature DCs, naïve T cells do not exhibit intrinsic alternate phases of stop and go [26, 31], suggesting that stop phases observed in vivo might rather result from constrains imposed by their environment. Nonetheless, the observation that Myosin IGdeficient $\mathrm{T}$ lymphocytes turn less frequently indicates that an actin-dependent intrinsic component might also be at work [36]. More recently, comparative modeling of $\mathrm{T}$ cell trajectories in lymph nodes has uncovered the heterogeneity of $\mathrm{T}$ cell behaviors in terms of speed, pauses and turns, while evolving in the same microenvironment [42, 43], suggesting that $\mathrm{T}$ cells in slightly different states may have different intrinsic migratory properties giving rise to distinct search strategies. Interestingly, neutrophil migration in response to laserinduced tissue damage can also be modeled by a Levy walk [43]. Beyond the specific choice of random walk model, neutrophils, just like DCs, display a stop and go behavior, which might optimize their chances to find and eliminate the microbes in tissues.

In summary, recent data advocate that leukocytes have rather optimized search strategies in vivo that are adapted to the density of targets and the tissue they navigate in. However, the contribution of stochastic cell-autonomous processes (such as an acto-myosin internal clock) and deterministic environmentally driven biases (such as neighboring cells or extracellular matrix) remains to be clarified for the different immune cell types. 


\section{ACTIN HELPS IMMUNE CELLS TO NAVIGATE IN A COMPLEX}

\section{ENVIRONMENT}

As highlighted in the introduction of this review, immune cells migrate in complex environments, which they can patrol randomly. Nonetheless, in some cases, they need some guidance to find their path: for example, when DCs become activated, they must reach lymphatic vessels to leave the tissues and migrate to lymph nodes in order to meet $\mathrm{T}$ lymphocytes. While chemokines have been thought for long to guide immune cells, it is only recently that the existence and the role of chemokine gradients have been formally demonstrated in vivo $[44,45]$. Similarly, although the concept of physical guidance of immune cells is well admitted [46], the individual contribution of the physical cues (confinement, stiffness, topology...) that guide them in vivo and the underlying mechanisms are far from being understood. Being guided requires two processes: the first one is to detect the guidance signal and the direction it indicates, and the second is to follow this direction by steering the migration path. Here, we will address the contribution of the actomyosin machinery to the guidance of immune cells following either chemical or physical guidance cues. We focus here on interstitial migration, but one should not forget that leukocyte crossing of mechanical barriers imposed by endothelial cells implicates specific mechanisms (during transmigration and diapedesis, via paracellular or transcellular routes $[47,48])$.

\section{The actomyosin cytoskeleton in chemical guidance of leukocytes}

Different mechanisms of chemotaxis have been proposed for leukocytes. While approaching the chemokine source they can increase: (1) their migration speed, a process referred to as orthotaxis, (2) their persistence, or (3) both parameters. Orthotaxis has recently been observed when analyzing the migration of neutrophils in zebrafish [45], as well as in mature DCs 
migrating in immobilized chemokine gradients in collagen gels or in the mouse skin [21, 44]. Interestingly, in the latter case, guidance is not controlled by Arp2/3, but rather by the formin mDia1, which is also needed for $\mathrm{T}$ lymphocyte chemotaxis [24, 49]. Although the mechanisms by which mDial controls the chemotactic response of DCs and T cells are not fully understood, the results obtained in both cell types are compatible with F-actin nucleation in response to chemokine stimulation being impaired. Formins might also play a role in the recently described "short-term directional memory of migrating HL-60 neutrophil-like cells" [50]. In this scenario, the presence of a chemokine gradient imposes the initial cell polarization and direction of migration, but migration persistence mostly results from directional memory. This would help leukocytes navigating in noisy environments. It might further provide an explanation for experiments showing that persistence of migration might be opposite to chemokine gradient when chemokines are released in waves. When the wave arrives to the front of the cell, it imposes cell polarization and the migration directionality. When the wave passes the cell, inverting the chemokine gradient along the cell axis, directional memory remains and keeps the cell moving forward. Nevertheless, prolonged exposure to a strong opposite gradient of chemokine can overcome this directional memory and force a cell to change direction.

Although, as discussed above, Arp2/3 activity is dispensable for the motility of immune cells in confinement, a recent study has highlighted the important role of the WAVE complex, which promotes Arp2/3-dependent actin nucleation at the cell front, in the chemotactic response of DCs [22]. On one hand, deletion of the WAVE gene renders immature DCs more persistent and faster, consistent with the results obtained with Arp2/3-deficient cells [21]. However, WAVE-deficient immature DCs have troubles turning in response to a chemokine gradient, even though their chemokinetic response (increase in speed in response to 
chemokine) remains unaltered. How WAVE contributes to such direction changes in immature DCs remains to be determined. On the other hand, in mature DCs, knocking out the WAVE gene results in the loss of the lamellipodium and appearance of filopodia. This compromises their ability to choose a direction as well as to find the pores for entering into lymphatic vessels, resulting in impaired migration of mature DCs to lymph nodes. These observations suggest that WAVE (and the resulting actin structures) might help sensing the environment and choosing an appropriate path. Whether the phenotype of WAVE-deficient DCs can be fully attributed to impaired Arp2/3 activation has not been shown. Interestingly, recent results obtained on Arp2/3-deficient macrophages suggest that it is only required for these cells to follow immobilized gradient of chemokines, an integrin-dependent process referred to as haptotaxis [27].

\section{The actomyosin cytoskeleton in leukocyte physical guidance}

While a lot of work has been focused on how cells interpret and follow chemokine gradients, it is only recently that efforts have been developed to evaluate the impact of physical cues on cell migration and guidance. This is particularly relevant for immune cells as, being highly migratory, they often have to cross tissues that exhibit different physical properties including rigidity, local topology, hydraulic resistance, etc...How do they adapt to the specific physical cues of the tissues they migrate through to be able to reach their final destination?

\section{Stiffness}

One physical property that varies greatly between different tissues and that can be sensed by cells, in general, is rigidity or stiffness [51]. Mechanosensing of global and local stiffness of the substrate during migration implicates the actomyosin contraction as well as integrindependent adhesion [52]. Generally, cells tend to migrate towards stiffer environments, a 
phenomenon referred to as durotaxis, which is associated to increased actomyosin contractility [53]. As in vivo interstitial migration of immune cells occurs independently of integrins [8], it is not clear whether these cells do sense the rigidity of their environment while evolving within tissues. Rigidity sensing through integrins might nonetheless play a role in situations where these molecules are involved, for example in migration along haptotactic chemokine gradients (see above), or in extravasation from vessels into tissues or lymphoid organs. In this context, it would be interesting to assess whether there are chemical or physical cues that are dominant over the other. For example, what does happen if a leukocyte simultaneously meets a soft-to-stiff and a chemokine gradient? Of note, it is also possible that immune cells have evolved to be insensitive to changes in the rigidity of their environment so that they can equally patrol tissues whether they are soft or stiff.

\section{Constrictions}

Another highly variable parameter within and between different tissues is porosity. Indeed, the organization of the ECM and the intercellular space of organs and membranes is such that immune cells often end up migrating in narrow spaces such as pores or confined channels. In vivo or in collagen gels, some immune cells can increase the porosity and dig their way through thanks to leukocyte proteases that degrade the ECM (such as neutrophil elastase, DC matrix-metalloproteases, cytotoxic $\mathrm{T}$ lymphocyte granzyme B). Yet, immune cells still encounter geometries exhibiting very small holes, experimentally in channels but also physiologically such as in the ear dermis [54]. In such geometries, the nucleus, as the stiffer organelle within the cell (due to the viscosity of chromatin and the presence of lamins), has been shown to be the main factor limiting cell locomotion [8, 55-57]. This effect can be used to design in vitro cell guiding assays $[58,59]$. Interestingly, the nuclei of different immune cell types do not exhibit the same size or the same deformability. This could enable or 
preclude different cell types from moving in tissues presenting small pores. The most striking example is neutrophils, which display multi-lobulated highly deformable nuclei and can therefore migrate in smaller holes than the majority of cells [55]. Interestingly, softness of neutrophil nuclei was shown to be more important than lobularity to squeeze through small constrictions [60]. How does the nucleus deform while cells migrate in confined spaces? Is nuclear deformation a passive consequence of pushing the nucleus through a small hole? Or is there a specific mechanism involved? We recently showed that when immature DCs migrate through constrictions (of $2 \mu \mathrm{m}$ width or below), a WAVE and Arp2/3-dependent cage of Factin assembles around their nucleus [61]. This actin cage induces nuclear lamin A breakage, making it more deformable, and enabling its passage through the constriction. This "confinement-induced actin network" might merely result from the response of the actin cortex to physical load [62], as it is also observed around stiff beads that have been engulfed by DCs and remain stuck within the constriction. Interestingly, HL60 neutrophil-like cells, which express low levels of lamin A and display a softer nucleus, can pass through small constrictions without Arp2/3 and with no detectable accumulation of actin. Consistent with these observations, DCs depleted of Lamin A/C do not need Arp2/3 anymore to pass the constrictions. Notably, the requirement of an actin cage for the nucleus to migrate in confined spaces has also been described for cancer cells [63] although in this case actin nucleation was dependent on Fascin interacting with the nuclear envelope protein Nesprin-2. These findings demonstrate the contribution of actin to the deformation of the nucleus and passage of a small constriction, but do not explain how the nucleus is pushed (or pulled?) through a narrow gap. Different studies, both in mature DCs [8] and in T cells [64, 65], highlighted the role of Myosin II in pushing the nucleus when a cell has to migrate through a constriction, either in collagen gels or in models of trans-endothelial migration. Surprisingly, neither immature DCs nor neutrophils need myosin II to migrate through channels with small constrictions, except in 
very small constrictions ( $1 \mu \mathrm{m}$ in width) for neutrophils, in which case the chromatin (which is viscous) could be the limiting element, requiring more force to pass through.

\section{Topography}

Each structure encountered by immune cells while migrating in tissues can present different nanoscale topographical organization, that could define some migration paths or on the contrary prevent migration in some places. The impact of topography on immune cell migration has been studied with $\mathrm{T}$ cells migrating on surfaces presenting nano-grooves, ridges or angles [66-68]. Different parameters influence $\mathrm{T}$ cell guidance by ridges, including the wavelength of the surface, as well as the value of angles between ridges, but also the level of confinement. The mechanisms contributing to $\mathrm{T}$ cell guidance by nanotopography have not been fully elucidated even though actin polymerization by Arp2/3, lamellipodium formation, and actomyosin contractility have been implicated in this process. It is also likely that microvilli or veiled structures present at the surface of leukocytes influence the guidance by nanotopographical structures, as the friction they generate will depend on the surface of the substrate. Altogether, these observations suggest that the impact of nanotopography can be very different depending on the confinement provided by the microenvironement, for example during interstitial migration versus extravasation. The precise nanotopographical structures that contribute to cell guidance in vivo have not been characterized so far.

\section{Hydraulic resistance}

Recently, hydraulic resistance has also emerged as a potential guiding clue for immune cells, leading to the concept of barotaxis [50]. When facing a bifurcation presenting two paths of distinct hydraulic resistance, neutrophils tend to choose the lower resistance path. They first extend arms in both directions, "testing" for resistance, resulting at some point in an 
asymmetry between the two extending arms, followed by the retraction of the shorter arm. The mechanism by which hydraulic resistance imposes cell directionality remains unknown. Notably, the physical cue provided by hydraulic resistance can overcome the chemical cue given by uncaging of a chemokine in front of the arm extending in high hydraulic resistance path. In this case, "chemical" polarization of the cell (as assessed by PH-Akt distribution) is reversed compared to chemotaxis, suggesting that distinct mechanisms control direction decision in cells depending on the nature of the guidance cue.

In summary, the pivotal role of actin and myosin II contractility in deciphering guidance cues and directing cell migration could help immune cells to prioritize the different cues and choose the best path so that they can efficiently navigate from one tissue to another.

\section{Concluding Remarks and Future Perspectives}

Here, we have presented multiple lines of evidence highlighting the essential role of the actomyosin cytoskeleton in the cell-intrinsic migratory properties of immune cells, as well as in their ability to respond to the chemical and physical cues present in the environment. It appears that different actin pools, which are generated by distinct actin nucleators and detected at distinct subcellular locations, exist in immune cells. Remarkably, these various actin pools are responsible for distinct cellular activities associated to cell migration. In DCs, $\mathrm{T}$ cells and neutrophils, the cortical actin pool localized towards the cell rear is nucleated by the formin mDial and responsible for forward movement in confinement. In contrast, the actin pool localized at their front is nucleated by Arp2/3, does not impact on migration but rather promotes cellular activities related to the individual functions of these cells: macropinocytosis in immature DCs, phagocytosis in neutrophils and immune synapse formation in $\mathrm{T}$ cells. Interestingly, this pool of actin imposes to leukocytes an intermittent migration mode that might be optimal for environment sampling. A third actin pool assembles 
around the nucleus of immature DCs and T cells as a result of confinement. This actin cage is also nucleated by Arp2/3 and helps deforming the nucleus for these cells to migrate through narrow pores. It is dispensable in neutrophils as they lack the Lamin $\mathrm{A} / \mathrm{C}$ proteins and thus have a softer nucleus as compared to DCs. Altogether, these findings suggest that compartmentalization of actin nucleating factors might play an essential role in leukocyte locomotion and guidance by physical external cues. How are actin dynamics regulated in space and time while immune cells migrate in their natural environment? How are environmental cues and thus leukocyte actin dynamics modified in pathology? Specifically, modifications of the extracellular matrix and interstitial fluid volume have been reported in inflammatory conditions [69]. The organization of the extracellular matrix directly influences porosity, rigidity or nanotopography of tissues, while the amount of interstitial fluid is likely to modify hydraulic resistance. Therefore, both of these parameters are expected to influence physical migration cues, and to reinforce or counteract chemical ones. Future experiments combining mouse intra-vital live imaging, tissue engineering and physical modeling should help addressing these questions in a quantitative manner (See Outstanding Questions).

\section{OUTSTANDING QUESTIONS BOX}

- What is the respective contribution of cell intrinsic properties and environment constraints in migration patterns exhibited by immune cells in vivo?

- How do cells integrate distinct environmental cues? Which cues (chemical or physical) are dominant on others?

- How is the environment modified in pathological situations? Are actin cytoskeleton properties still contributing to optimized immune cell function in these situations? 


\section{Acknowledgements}

This work was supported by grants from the Association pour la Recherche contre le Cancer (ARC-PDF20140601095 to HDM) and the DCBIOL Labex (ANR-10-IDEX-0001-02-PSL and ANR-11-LABX-0043) to A.M.L.-D., as well as the ANR (PhyMax), the Fondation pour la recherché Médicale and the Institut National du Cancer to A.-M.L.-D., M.P. and R.V. Conflict of interest statement: The authors declare no conflict of interest.

\section{TEXT BOX: Which strategy is the best for immune cells looking for a target? Intermittent} search strategy versus Levy walks models.

Different theoretical models have been used to characterize immune cell trajectories and to estimate their efficiency at finding a target. These studies emphasize the difficulty of identifying the mode of leukocyte migration that gives the best trade-offs over the numerous parameters characterizing migration, but also highlight the power of modeling for formulation of new hypotheses on intrinsic versus extrinsic migratory properties of immune cells. Regarding target finding, the two main models used are "intermittent search strategy", which alternates fast migration with limited environment sampling and slow migration with sampling, and "Levy walks", which consists of a succession of randomly oriented persistent runs, whose lengths are broadly distributed. Both have been shown theoretically to efficiently explore space. In practice, Levy walks share a similar stop and go behavior with intermittent random walks, and their experimental identification is often disputed. The main difference relies on the hypothesis that target detection (e.g. antigen uptake in the case of DCs) is more efficient during pauses in intermittent random walks, whereas it is assumed independent of cell speed in Levy walks. 


\section{References}

1. Danuser, G., J. Allard, and A. Mogilner, Mathematical modeling of eukaryotic cell migration: insights beyond experiments. Annu Rev Cell Dev Biol, 2013. 29: p. 50128.

2. Juelicher, F., et al., Active behavior of the cytoskeleton. Physics Reports, 2007. 449(1): p. 3-28.

3. Prost, J., F. Juelicher, and J.-F. Joanny, Active gel physics. Nature Physics, 2015. 11: p. 111-117.

4. Sanz-Moreno, V., et al., Rac activation and inactivation control plasticity of tumor cell movement. Cell, 2008. 135(3): p. 510-23.

5. Callan-Jones, A.C. and R. Voituriez, Actin flows in cell migration: from locomotion and polarity to trajectories. Curr Opin Cell Biol, 2016. 38: p. 12-7.

6. Paluch, E.K., I.M. Aspalter, and M. Sixt, Focal Adhesion-Independent Cell Migration. Annu Rev Cell Dev Biol, 2016. 32: p. 469-490.

7. Malawista, S.E. and A. de Boisfleury Chevance, Random locomotion and chemotaxis of human blood polymorphonuclear leukocytes (PMN) in the presence of EDTA: PMN in close quarters require neither leukocyte integrins nor external divalent cations. Proc Natl Acad Sci U S A, 1997. 94(21): p. 11577-82.

8. Lammermann, T., et al., Rapid leukocyte migration by integrin-independent flowing and squeezing. Nature, 2008. 453(7191): p. 51-5.

9. Renkawitz, J., et al., Adaptive force transmission in amoeboid cell migration. Nat Cell Biol, 2009. 11(12): p. 1438-43.

10. Liu, Y.J., et al., Confinement and low adhesion induce fast amoeboid migration of slow mesenchymal cells. Cell, 2015. 160(4): p. 659-72.

11. Bergert, M., et al., Force transmission during adhesion-independent migration. Nat Cell Biol, 2015. 17(4): p. 524-9.

12. Ruprecht, V., et al., Cortical contractility triggers a stochastic switch to fast amoeboid cell motility. Cell, 2015. 160(4): p. 673-85. 
13. Overstreet, M.G., et al., Inflammation-induced interstitial migration of effector CD4(+) T cells is dependent on integrin alphaV. Nat Immunol, 2013. 14(9): p. 94958.

14. von Andrian, U.H., et al., A central role for microvillous receptor presentation in leukocyte adhesion under flow. Cell, 1995. 82(6): p. 989-99.

15. Hawkins, R.J., et al., Pushing off the walls: a mechanism of cell motility in confinement. Phys Rev Lett, 2009. 102(5): p. 058103.

16. Friedl, P., et al., CD4+ T lymphocytes migrating in three-dimensional collagen lattices lack focal adhesions and utilize beta1 integrin-independent strategies for polarization, interaction with collagen fibers and locomotion. Eur J Immunol, 1998. 28(8): p. 2331-43.

17. Maiuri, P., et al., Actin flows mediate a universal coupling between cell speed and cell persistence. Cell, 2015. 161(2): p. 374-86.

18. Hawkins, R.J., et al., Spontaneous contractility-mediated cortical flow generates cell migration in three-dimensional environments. Biophys J, 2011. 101(5): p. 1041-5.

19. Callan-Jones, A.C. and R. Voituriez, Active Gel Model of Amoeboid Cell Motility. New Journal of Physics, 2013. 15.

20. Abu Shah, E. and K. Keren, Symmetry breaking in reconstituted actin cortices. Elife, 2014. 3: p. e01433.

21. Vargas, P., et al., Innate control of actin nucleation determines two distinct migration behaviours in dendritic cells. Nat Cell Biol, 2016. 18(1): p. 43-53.

22. Leithner, A., et al., Diversified actin protrusions promote environmental exploration but are dispensable for locomotion of leukocytes. Nat Cell Biol, 2016. 18(11): p. 1253-1259.

23. Wilson, K., et al., Mechanisms of leading edge protrusion in interstitial migration. Nat Commun, 2013. 4: p. 2896.

24. Eisenmann, K.M., et al., $T$ cell responses in mammalian diaphanous-related formin mDia1 knock-out mice. J Biol Chem, 2007. 282(34): p. 25152-8.

25. Tanizaki, H., et al., Rho-mDia1 pathway is required for adhesion, migration, and Tcell stimulation in dendritic cells. Blood, 2010. 116(26): p. 5875-84.

26. Moreau, H.D., et al., Signal strength regulates antigen-mediated T-cell deceleration by distinct mechanisms to promote local exploration or arrest. Proc Natl Acad Sci U S A, 2015. 112(39): p. 12151-6. 
27. Rotty, J.D., et al., Arp2/3 Complex Is Required for Macrophage Integrin Functions but Is Dispensable for FcR Phagocytosis and In Vivo Motility. Dev Cell, 2017. 42(5): p. 498-513 e6.

28. Chabaud, M., et al., Cell migration and antigen capture are antagonistic processes coupled by myosin II in dendritic cells. Nat Commun, 2015. 6: p. 7526.

29. Faure-Andre, G., et al., Regulation of dendritic cell migration by CD74, the MHC class II-associated invariant chain. Science, 2008. 322(5908): p. 1705-10.

30. Lavi, I., et al., Deterministic patterns in cell motility. Nature Physics, 2016. 12: p. 1146-1152.

31. Jacobelli, J., et al., Confinement-optimized three-dimensional $T$ cell amoeboid motility is modulated via myosin IIA-regulated adhesions. Nat Immunol, 2010. 11(10): p. 953-61.

32. Friedman, R.S., J. Jacobelli, and M.F. Krummel, Surface-bound chemokines capture and prime T cells for synapse formation. Nat Immunol, 2006. 7(10): p. 1101-8.

33. Andzelm, M.M., et al., Myosin IIA is required for cytolytic granule exocytosis in human NK cells. J Exp Med, 2007. 204(10): p. 2285-91.

34. Jacobelli, J., et al., Myosin-IIA and ICAM-1 regulate the interchange between two distinct modes of T cell migration. J Immunol, 2009. 182(4): p. 2041-50.

35. Vascotto, F., et al., The actin-based motor protein myosin II regulates MHC class II trafficking and BCR-driven antigen presentation. J Cell Biol, 2007. 176(7): p. 100719.

36. Gerard, A., et al., Detection of rare antigen-presenting cells through T cell-intrinsic meandering motility, mediated by Myo1g. Cell, 2014. 158(3): p. 492-505.

37. Santos-Argumedo, L., J.L. Maravillas-Montero, and O. Lopez-Ortega, Class I myosins in B-cell physiology: functions in spreading, immune synapses, motility, and vesicular traffic. Immunol Rev, 2013. 256(1): p. 190-202.

38. Miller, M.J., et al., Autonomous T cell trafficking examined in vivo with intravital two-photon microscopy. Proc Natl Acad Sci U S A, 2003. 100(5): p. 2604-9.

39. Harris, T.H., et al., Generalized Levy walks and the role of chemokines in migration of effector CD8+ T cells. Nature, 2012. 486(7404): p. 545-8.

40. Beltman, J.B., et al., Lymph node topology dictates T cell migration behavior. J Exp Med, 2007. 204(4): p. 771-80. 
41. Dustin, M.L., Stop and go traffic to tune T cell responses. Immunity, 2004. 21(3): p. 305-14.

42. Banigan, E.J., et al., Heterogeneous CD8+ T cell migration in the lymph node in the absence of inflammation revealed by quantitative migration analysis. PLoS Comput Biol, 2015. 11(2): p. e1004058.

43. Read, M.N., et al., Leukocyte Motility Models Assessed through Simulation and Multi-objective Optimization-Based Model Selection. PLoS Comput Biol, 2016. 12(9): p. e1005082.

44. Weber, M., et al., Interstitial dendritic cell guidance by haptotactic chemokine gradients. Science, 2013. 339(6117): p. 328-32.

45. Sarris, M., et al., Inflammatory chemokines direct and restrict leukocyte migration within live tissues as glycan-bound gradients. Curr Biol, 2012. 22(24): p. 2375-82.

46. Charras, G. and E. Sahai, Physical influences of the extracellular environment on cell migration. Nat Rev Mol Cell Biol, 2014. 15(12): p. 813-24.

47. Carman, C.V. and R. Martinelli, T Lymphocyte-Endothelial Interactions: Emerging Understanding of Trafficking and Antigen-Specific Immunity. Front Immunol, 2015. 6: p. 603.

48. Filippi, M.D., Mechanism of Diapedesis: Importance of the Transcellular Route. Adv Immunol, 2016. 129: p. 25-53.

49. Sakata, D., et al., Impaired T lymphocyte trafficking in mice deficient in an actinnucleating protein, mDia1. J Exp Med, 2007. 204(9): p. 2031-8.

50. Prentice-Mott, H.V., et al., Biased migration of confined neutrophil-like cells in asymmetric hydraulic environments. Proc Natl Acad Sci U S A, 2013. 110(52): p. 21006-11.

51. Discher, D.E., P. Janmey, and Y.L. Wang, Tissue cells feel and respond to the stiffness of their substrate. Science, 2005. 310(5751): p. 1139-43.

52. Pasapera, A.M., et al., Rac1-dependent phosphorylation and focal adhesion recruitment of myosin IIA regulates migration and mechanosensing. Curr Biol, 2015. 25(2): p. 175-86.

53. Trichet, L., et al., Evidence of a large-scale mechanosensing mechanism for cellular adaptation to substrate stiffness. Proc Natl Acad Sci U S A, 2012. 109(18): p. 69338. 
54. Raab, M., et al., ESCRT III repairs nuclear envelope ruptures during cell migration to limit DNA damage and cell death. Science, 2016. 352(6283): p. 359-62.

55. Wolf, K., et al., Physical limits of cell migration: control by ECM space and nuclear deformation and tuning by proteolysis and traction force. J Cell Biol, 2013. 201(7): p. 1069-84.

56. Harada, T., et al., Nuclear lamin stiffness is a barrier to 3D migration, but softness can limit survival. J Cell Biol, 2014. 204(5): p. 669-82.

57. Davidson, P.M., et al., Design of a microfluidic device to quantify dynamic intranuclear deformation during cell migration through confining environments. Integr Biol (Camb), 2015. 7(12): p. 1534-46.

58. Caballero, D., et al., Ratchetaxis: Long-Range Directed Cell Migration by Local Cues. Trends Cell Biol, 2015. 25(12): p. 815-27.

59. Le Berre, M., et al., Geometric Friction Directs Cell Migration. Phys Rev Lett, 2013. 111(19): p. 198101.

60. Rowat, A.C., et al., Nuclear envelope composition determines the ability of neutrophil-type cells to passage through micron-scale constrictions. J Biol Chem, 2013. 288(12): p. 8610-8.

61. Thiam, H.R., et al., Perinuclear Arp2/3-driven actin polymerization enables nuclear deformation to facilitate cell migration through complex environments. Nat Commun, 2016. 7: p. 10997.

62. Bieling, P., et al., Force Feedback Controls Motor Activity and Mechanical Properties of Self-Assembling Branched Actin Networks. Cell, 2016. 164(1-2): p. 115-127.

63. Jayo, A., et al., Fascin Regulates Nuclear Movement and Deformation in Migrating Cells. Dev Cell, 2016. 38(4): p. 371-83.

64. Soriano, S.F., et al., In vivo analysis of uropod function during physiological T cell trafficking. J Immunol, 2011. 187(5): p. 2356-64.

65. Jacobelli, J., et al., Activated T cell trans-endothelial migration relies on myosin-IIA contractility for squeezing the cell nucleus through endothelial cell barriers. PLoS One, 2013. 8(9): p. e75151.

66. Song, K.H., et al., Sinusoidal wavy surfaces for curvature-guided migration of $T$ lymphocytes. Biomaterials, 2015. 51: p. 151-60.

67. Kwon, K.W., et al., Nanotopography-guided migration of T cells. J Immunol, 2012. 189(5): p. 2266-73. 
68. Kwon, K.W., H. Park, and J. Doh, Migration of T cells on surfaces containing complex nanotopography. PLoS One, 2013. 8(9): p. e73960.

69. Kai, F., H. Laklai, and V.M. Weaver, Force Matters: Biomechanical Regulation of Cell Invasion and Migration in Disease. Trends Cell Biol, 2016. 26(7): p. 486-497. 


\section{FIGURE LEGENDS}

Figure 1: Actin, forces and non-adherent migration under confinement. Myosin II accumulation at the back of the cell maintains the actin retrograde flow, which generates unspecific friction between the cortex and the environment allowing momentum transfer. This results in effective non-adherent migration, even in environments preventing any adhesion as long as confinement can provide some level of friction.

Figure 2: Actomyosin control of migratory patterns of immune cells. In dendritic cells, formin-mediated cabled actin polymerization at the rear of the cell results in fast directional migration. This is reinforced by myosin accumulation at the back by Invariant chain leading to higher contractility at the rear. On the contrary, Arp2/3-mediated branched actin together with Myosin II and Ii at the front of the cell is associated with slow migration and environment sampling by macropinocytosis. Alternance of these two types of migration (whether it is cell intrinsic or environment driven) optimizes search strategy of the environment. Interestingly similar cytoskeleton and migration behaviors have been reported for other immune cells such as T cells of NK cells.

Figure 3: Actomyosin cytoskeleton in leukocyte guidance. Immune cells are guided by a combination of chemical and physical extracellular cues. Actin is highly implicated in detection and response to these guidance cues. Having actin as a central player can enable cells to integrate the different cues and choose their global direction of migration in complex environments. 
Confinement can provide friction in absence of adhesion

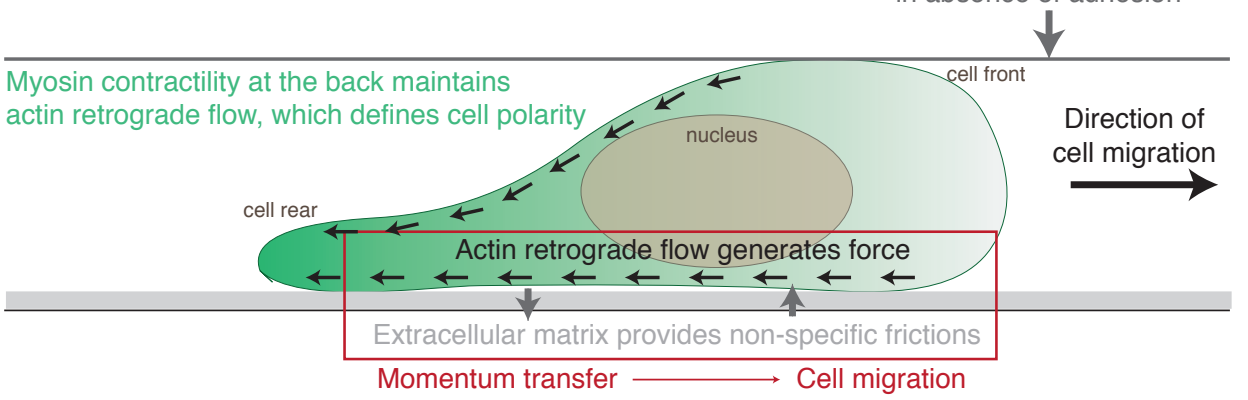


Moreau et al, Figure 2

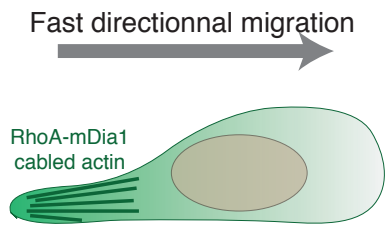

Acto-myosin gradient

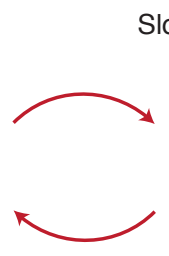

Slow migration and

Macropinocytosis (DCs) Immune synapse ( $B$ and $T$ cells) Killing synapse (NK and T cells)

Cdc42-Arp2/3

branched actin

Alternating fast and slow migration

optimizes search strategy 
Moreau et al, Figure 3

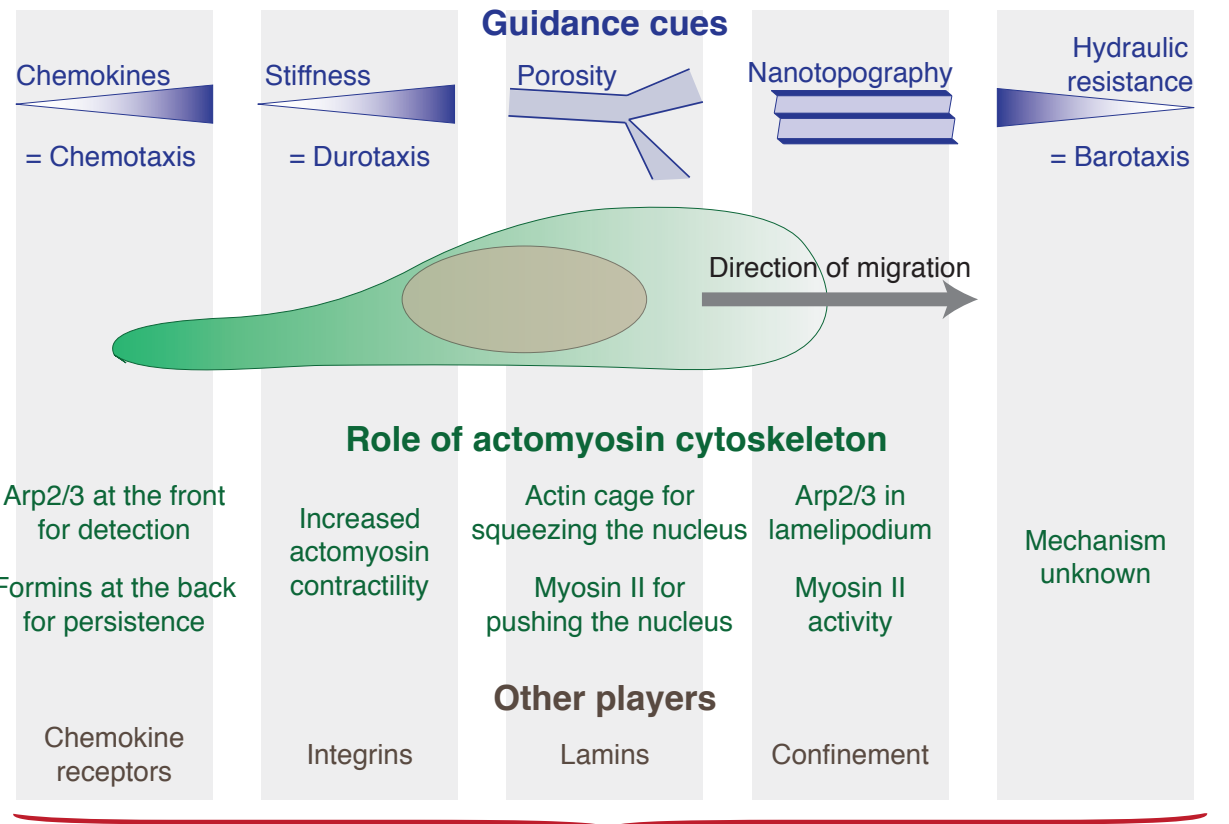

Integrated migratory response to complex environmental guidance cues 